\title{
Temporal Variation in Staphylococcus aureus Protein A Genotypes from Nose and Skin in Atopic Dermatitis Patients
}

\author{
Minke M.F. van Mierlo ${ }^{a}$ Suzanne G.M.A. Pasmans ${ }^{a}$ Joan E.E. Tottéa,b \\ Jill de Wit ${ }^{a}$ Bjorn L. Herpers ${ }^{c}$ Margreet C. Vos ${ }^{d}$ Corné H.W. Klaassen ${ }^{d}$ \\ Luba M. Pardo ${ }^{a}$ \\ a Department of Dermatology, Erasmus MC University Medical Center Rotterdam-Sophia Children's Hospital- \\ Center of Pediatric Dermatology, Rotterdam, The Netherlands; ${ }^{b}$ Department of Medical Microbiology, University \\ Medical Center Utrecht, Utrecht, The Netherlands; ' ${ }^{C}$ Regional Public Health Laboratory Kennemerland, Haarlem, The \\ Netherlands; ${ }^{d}$ Department of Medical Microbiology and Infectious Diseases, Erasmus MC University Medical Center \\ Rotterdam, Rotterdam, The Netherlands
}

\section{Keywords}

Atopic dermatitis - Eczema $\cdot$ Microbiome $\cdot$ Staphylococcus aureus

\section{Abstract}

Background: Staphylococcus aureus colonization is associated with disease severity in patients with atopic dermatitis (AD). Objective: To investigate temporal variation in S. aureus protein A gene (spa)-types isolated from the nose and lesional skin and the correlation of spa-types with disease severity. Results: This study included 96 adult AD patients who were assessed at baseline (T0) and after a strict 2-week follow-up period (T1) in which treatment was standardized with a topical corticosteroid. Fifty-five different spa-types were detected in the nose and skin cultures. Seventy-three patients were colonized with S. aureus in the nasal cavity at both time points (persistent carriership), 59 of whom (81\%) had identical spa-types over time. For skin samples, 42 (75\%) of the 56 persistent skin carriers had identical spa-types over time. The same spa-type was carried in the nose and skin in 79 and $77 \%$ of the patients at T0 and T1, respectively. More severe disease was not associated with specific spa-types or with temporal variation in spa-type. Conclusion: S. aureus strains in AD are highly heterogeneous between patients. The majority of patients carry the same spa-type in the nose and skin without temporal variation, suggesting clonal colonization within individual patients. No predominant spatype or temporal variation is associated with increased disease severity.

(C) 2021 The Author(s)

Published by S. Karger AG, Basel

\section{Introduction}

Atopic dermatitis (AD) is the most common inflammatory skin disease with a lifetime prevalence of $15-20 \%$ and a significant negative impact on quality of life [1]. The pathogenesis of AD is multifactorial, involving skin barrier defects and immunological dysregulation [2]. Moreover, strong evidence links the skin microbiome, characterized by an increased abundance of Staphylococcus aureus and less bacterial diversity, to the pathogenesis and disease severity in this group of patients [2-6].

karger@karger.com www.karger.com/drm

Karger $\stackrel{\text { ' }}{5}$

BOPEN ACCESS
(C) 2021 The Author(s)

Published by S. Karger AG, Basel

This is an Open Access article licensed under the Creative Commons Attribution-NonCommercial-4.0 International License (CC BY-NC) (http://www.karger.com/Services/OpenAccessLicense), applicable to the online version of the article only. Usage and distribution for commercial purposes requires written permission.
Correspondence to:

Minke M.F. van Mierlo, m.vanmierlo@erasmusmc.nl

Luba Milena Pardo, l.pardocortes@erasmusmc.nl 
S. aureus is both a human commensal organism and a pathogen [7]. The most frequent carriage site is the nose, which is assumed to be the main reservoir for the spread of the pathogen associated with skin infections $[7,8]$. Approximately $20 \%$ of the general population is a persistent carrier of S. aureus in the nose [9]. Cross-sectional data on $\mathrm{AD}$ patients show a prevalence of $62 \%$ in nasal $S$. aureus colonization and $70 \%$ on the lesional skin [3].

The association between $S$. aureus colonization and disease severity has been thoroughly investigated, but the underlying mechanism resulting in the characteristic $\mathrm{AD}$ inflammation remains unclear [10]. One of the difficulties in understanding the role of $S$. aureus in $\mathrm{AD}$ is that this bacterium is a common colonizer (in nose and on the skin). When $S$. aureus is present on the skin, it is difficult to understand which specific strains can cause inflammation as it can carry a wide diversity of virulence factors enabling transmission, virulence, and antibiotic resistance [11]. It was shown that the type of inflammation and immune signature characteristic of AD depends on the S. aureus strain [12]. Furthermore, it has been proposed that temporal variation in colonizing the $S$. aureus strain is an aggravating factor in $\mathrm{AD}$ due to a new trigger that is introduced to the immune system $[13,14]$. This emphasizes that strain differences may contribute to the complexity of the disease, encouraging a more extensive investigation into the $S$. aureus populations in $\mathrm{AD}$.

Multiple molecular identification methods are available for discriminating among $S$. aureus isolates. A singlelocus typing technique, spa-typing, offers a subtyping resolution comparable to more expensive and/or laborious techniques such as multilocus sequence typing (MLST) and pulsed field gel electrophoresis (PFGE) [15]. The spatyping technique targets the polymorphic region of the $S$. aureus-specific staphylococcal protein A gene $(s p a)$. The encoding region consists of a variable number of repeats with highly variable nucleotide sequences [16]. Since it is sequence-based, the results produced in different labs are easily comparable.

Literature on the temporal variation of S. aureus and the association with disease severity is scarce. Due to low patient numbers with multiple treatment regimens and the absence of a strict follow-up time, results are subject to cofounders $[13,14,17]$. This study investigates the temporal variation in $S$. aureus spa-types in adult $\mathrm{AD}$ patients with a strict follow-up period of 2 weeks and standardized treatment, which reduced possible confounding of the comparisons over time. Furthermore, we assess similarities in spa-types from the nose and skin and cor- relations with disease severity. Knowledge of the colonization patterns of $S$. aureus in AD might help us to develop new strategies to improve inflammation.

\section{Material and Methods}

\section{Study Design}

This was a longitudinal study embedded in the MAAS trial (ClinicalTrials.gov NCT02840955). Details of the clinical trial and primary outcomes have been published previously $[18,19]$. We included measurements from time point 0 (T0, baseline) and time point 1 (T1). T1 was scheduled after a 2 -week run-in period, in which treatment was standardized with triamcinoloneacetonide $0.1 \%$ cream according to a corticosteroid dosing regimen.

\section{Study Population}

Adult patients ( $\geq 18$ years) with moderate to severe AD, according to the UK Working Party diagnostic criteria for $\mathrm{AD}$, defined by an Eczema Area and Severity Index (EASI) score of 7.150.0 , were eligible for participation in the study. Other inclusion criteria were the use of any topical corticosteroid (TCS) before enrolment and ability to read and understand the patient information. Patients were ineligible for participation if they had used systemic antibiotics or systemic corticosteroids in the previous 2 months, oral immunosuppressive agents or ultraviolet light therapy in the previous 3 months, or topical antibiotics in the previous week. Another exclusion criterion was the presence of another skin condition that could interfere with the assessment of the AD severity. Patients were characterized by demographic data, and disease severity was assessed at both time points by the research physician using the EASI score. Based on the continuous EASI score, patients were categorized as having almost-clear, mild, moderate, or severe disease, as described previously [20]. This study included patients from whom bacterial cultures were collected at both time points.

\section{Sample Collection and spa-Typing}

Samples for culture analysis were collected using sterile dry Copan 490CE swabs, which were transferred into liquid Amies medium after collection. Skin swabs were collected from the lesional skin, preferably located at the antecubital fold or the popliteal fold at T0. These lesions did not show signs of impetigo. At T1, the skin swab was collected from the location chosen at T0. Nose swabs were collected from both the anterior nares at T0 and T1.

Bacterial semiquantitative cultures were performed using routine diagnostic culture procedures, blood agar plates, and specific $S$. aureus culture plates (ChromID S. aureus Elite agar [SAIDE], Biomérieux, France). Plates were incubated at $35^{\circ} \mathrm{C}$ and growth was assessed after 1 and 2 days of culture. Subsequent species determination by MALDI-TOF (Bruker Daltonics, Bremen, Germany) was performed. For each distinct colony morphology of $S$. aureus in a sample, a representative colony was picked to store the strain at $-20^{\circ} \mathrm{C}$ for future analysis. The semiquantitative $S$. aureus cultures were reported on a categorical scale ranging from 0 (negative) to 4 (strongly positive).

DNA was extracted from the collected $S$. aureus isolates using a MagNA Pure 96 platform in combination with the MagNA Pure DNA and Viral Nucleic Acid Small Volume Kit (Roche Diagnostics, Almere, The Netherlands). PCR reactions were performed in 
$25-\mu \mathrm{L}$ reactions using $1 \mu \mathrm{L}$ of isolated DNA in $1 \times$ FastStart PCR Master (Roche) and $0.5 \mu \mathrm{M}$ of both forward primer ( $5^{\prime}$-AACAACGTAACGGCTTCATCC- $3^{\prime}$ ) and reverse primer (5'-GCTTTTGCAATGTCATTTACTG-3'). Thermal cycling consisted of an initial denaturation step for $10 \mathrm{~min}$ at $95^{\circ} \mathrm{C}$, followed by 35 cycles of $30 \mathrm{~s}$ at $95^{\circ} \mathrm{C}, 30 \mathrm{~s}$ at $60^{\circ} \mathrm{C}$, and $1 \mathrm{~min}$ at $72^{\circ} \mathrm{C}$. After a final extension step of $10 \mathrm{~min}$ at $72^{\circ} \mathrm{C}$, reactions were cooled to room temperature. Five microliters of PCR product were analyzed on agarose gel to confirm amplification. The remainder of the PCR product was treated with $2 \mu \mathrm{L}$ of ExoSAP-IT (Isogen Life Science, De Meern, The Netherlands) for $15 \mathrm{~min}$ at $37^{\circ} \mathrm{C}$ following inactivation for $15 \mathrm{~min}$ at $80^{\circ} \mathrm{C}$. Amplicon sequencing was performed by BaseClear (Leiden, The Netherlands) using the forward amplification primer as a sequencing primer. Electropherograms were analyzed and interpreted using the spa-typing plugin in BioNumerics software v7.6 (Applied Maths, Sint-Martens-Latem, Belgium).

\section{Statistical Analysis}

Descriptive statistics were used to present patient and sample characteristics. Persistent nose and skin carriers were defined as patients with a positive $S$. aureus culture at both time points for the nose and skin, respectively. Intermittent carriers were defined as having a positive $S$. aureus culture at only 1 time point (T0 or T1), either in the nose or skin. For the difference in EASI score over time, the Wilcoxon signed rank test was used. The Mann-Whitney $\mathrm{U}$ test was performed to determine the differences in EASI score for $S$. aureus colonization and temporal variation in spa-type. The $S$. aureus load, as determined by semiquantitative culture, between both time points was tested using the $\chi^{2}$ test. Statistical analysis was performed in SPSS v25. $p \leq 0.05$ was considered statistically significant.

\section{Results}

\section{Population Characteristics}

A total of 100 patients were eligible for participation in this study. Bacterial cultures were available from 96 patients at both time points and thus included in this study. The median age was 32.0 years (interquartile range [IQR] 25.0-47.0) and 45 (47\%) patients were female. The median EASI score at T0 was 13.1 (IQR 9.4-19.0), compared to $8.1(5.0-13.8)$ after the 2 -week run-in period with triamcinolone $(p<0.01$; Table 1$)$. We found high colonization rates in both the nose and skin at both time points (Table 1; Fig. 1).

\section{Heterogeneous S. aureus among AD Patients with \\ Identical Spa-Types in the Nose and Skin}

Of the 290 S. aureus-positive cultures (nose T0: 79; nose T1: 78; skin T0: 71; skin T1: 62), 285 isolates were available for spa-typing. All $S$. aureus-positive cultures contained only 1 distinct morphological colony type of $S$. aureus. Fifty-five different spa-types were found, with t002 (10\%), t091 (8\%), t127 (8\%), t571 (7\%), and t189
Table 1. Characteristics of 96 patients

$\begin{array}{lc}\text { Age, years } & 32.0(25.0-47.0) \\ \text { Female } & 45(46.9) \\ \text { Race } & \\ \quad \text { American or Alaska-Native } & 5(5.2) \\ \quad \text { Asian } & 10(10.4) \\ \quad \text { Black or African-American } & 7(7.3) \\ \text { White } & 74(77.1) \\ \text { EASI }{ }^{1} & \\ \text { T0 } & 13.1(9.4-19.0) \\ \text { T1 } & 8.1(5.0-13.8) \\ \text { Positive for S. aureus } \text { nose }^{2} & \\ \text { T0 } & 79(82.3) \\ \text { T1 } & 78(81.3) \\ \text { Positive for S. aureus } \text { skin }^{3} & \\ \text { T0 } & 71(74.0) \\ \text { T1 } & 62(64.6)\end{array}$

Values are expressed as $n(\%)$ or median (IQR). EASI, eczema area and severity index; T0, baseline; T1, 2 weeks after baseline.

${ }^{1}$ The EASI score ranges from 0 to 72 .

$273(76.0 \%)$ patients were colonized in the nasal cavity at both time points.

$356(58.3 \%)$ patients were colonized on the skin at both time points.

(6\%) being the most common, accounting for $<40 \%$ of all spa-types. Figure 2 shows the prevalence of the 10 most common spa-types separated for the nose and skin. The total of 55 spa-types is depicted in online supplementary Table 1 and Figure 1 (for all online suppl. material, see www.karger.com/doi/10.1159/000515235). Furthermore, online supplementary Table 1 shows that the spatypes found in our study population are also present in previously investigated $\mathrm{AD}$ populations and within the general population [21-26].

Sixty-six patients had positive $S$. aureus cultures in both the nose and skin at baseline, including 79\% (52/66) with identical spa-types at both locations. At T1, the group of patients positive at both sample locations included 59 patients, 46 of whom (78\%) carried the same spa-type.

High Prevalence of Persistent S. aureus Carriers in the Nose and Skin with Identical spa-Types

Our study population comprised 76\% (73/96) persistent and 11\% (11/96) intermittent nose carriers. Eighty percent $(59 / 73)$ of the persistent nose carriers were colonized with the same spa-type during follow-up. On AD skin, 58\% (56/96) patients were persistent carriers and $22 \%(21 / 96)$ intermittent carriers. In the group of persis- 
Fig. 1. Temporal $S$. aureus colonization. Of the 84 patients positive at baseline, 66 (79\%) were positive in both nose and skin, $13(15 \%)$ only in the nose, and 5 (6\%) only on the skin. Of the 81 patients positive at T1, 59 (73\%) were positive for both nose and skin, 19 (23\%) only in the nose, and 3 (4\%) only on the skin.

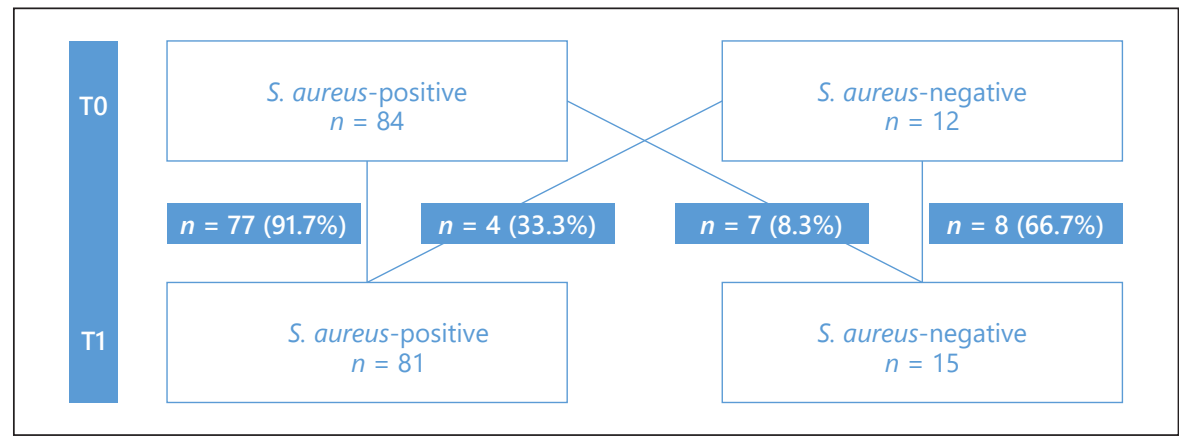

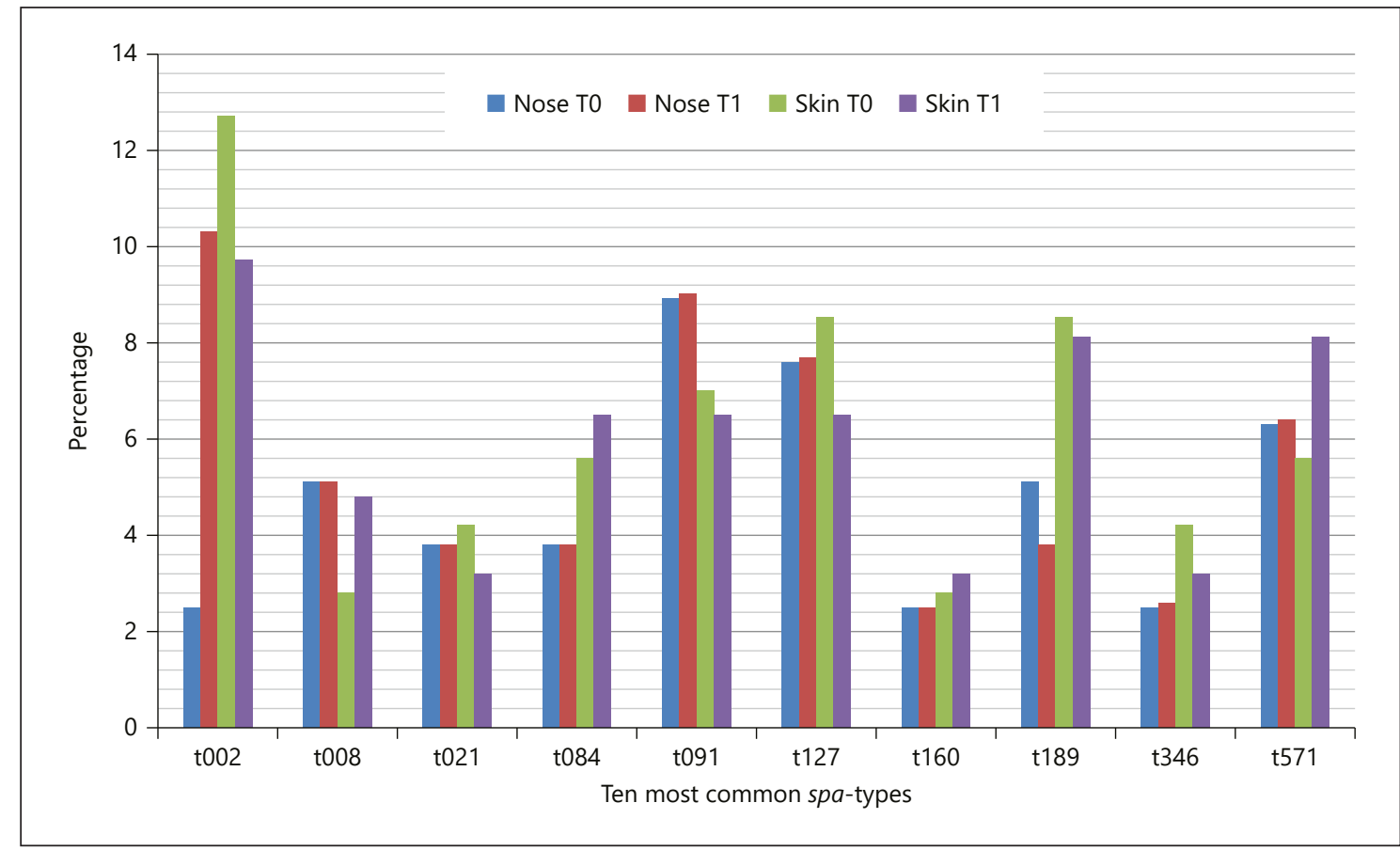

Fig. 2. Frequency of the 10 most common spa-types per location and time point. The 10 most common spa-types in the nose samples at T0 represent $48.1 \%$ of the total spa-types. For the nose samples at T1, the 10 most common spa-types represented $53.8 \%$ of the total. For the skin samples at T0 and T1, this was 61.9 and $59.8 \%$, respectively. The overview of all spa-types is presented in online supplementary Figure 1.

tent skin carriers, 79\% (44/56) of the patients had identical spa-types over time.

\section{Disease Severity Is Not Associated with the spa-Type}

Present in the Nose and Skin

Patients who were positive for S. aureus (nose and/or skin) at baseline had a significantly higher EASI score at T0 than those who were negative for $S$. aureus $(p=0.02)$. The median EASI score was 14.2 (IQR 9.9-19.2) and 9.1 (IQR 7.7-14.8), respectively. At T1, after the standardized 2-week run-in treatment with triamcinolone, there was no difference in EASI score anymore between S. aureuspositive and $S$. aureus-negative patients (EASI 8.5 [IQR 5.0-14.4] and 8.0 [IQR 4.8-12.0], respectively; $p=0.55$ ). Interestingly, the EASI score and the $S$. aureus density in the nose and skin at T1 was significantly lower than at T0 (Table 1; online suppl. Table 2). Looking into the abundance of the 5 most common spa-types per severity category, we did not find an overrepresentation of a certain spa-type (online suppl. Fig. 2). Because of the high num- 
ber of spa-types with a low prevalence and the fact that these numbers are accounted for by only a few patients, this was not amenable for statistical testing. Between patients with and without a change in spa-type over time (in the nose and/or skin), the change in EASI score from T0 to T1 did not differ significantly ( $p=0.11$; online suppl. Fig. 3).

\section{Discussion}

Our study showed a heterogeneous $S$. aureus population in $\mathrm{AD}$ patients, as seen in the general population. Spa-types $\mathrm{t} 002, \mathrm{t} 091, \mathrm{t} 127, \mathrm{t} 571$, and $\mathrm{t} 189$ were the most common and the remaining types were almost unique to 1 patient. Large proportion of the patients $(79 \%$ at $\mathrm{T} 0$ and $78 \%$ at $\mathrm{T} 1$ ) had positive cultures in both the nose and skin and were colonized with only 1 spa-type. Eighty percent (59/73) of the persistent nose carriers were colonized with the same spa-type compared to $79 \%$ (44/56) of the persistent skin carriers. Lastly, we showed that disease severity was not affected by the spa-type present or by a change in spa-type over time.

Our results are in line with previous publications describing a heterogeneous population structure of $S$. aureus in $\mathrm{AD}$ and the absence of a prevailing strain $[14,21$, $22]$. Moreover, the most common spa-types found in our study population are also present in previously investigated $\mathrm{AD}$ populations and the general population, although the prevalence point estimates are not the same [14, 21-27]. This study confirmed that there are no specific spa-types preferentially colonizing patients with $\mathrm{AD}$ compared to healthy individuals. It could be argued that spa-typing does not have enough resolution to identify the genetic diversity between strains in $\mathrm{AD}$. Compared to whole-genome sequencing (WGS) techniques, that examine complete genomes, spa-typing is based on a single gene [28]. However, WGS remains expensive to conduct in large series of cases and the spa-typing technique has proven successful in settings requiring high levels of discrimination $[28,29]$.

The nose is commonly described as the most important reservoir for $S$. aureus and several studies show identical $S$. aureus strains in the nose and infected skin regions $[7,30,31]$. Moreover, nasal eradication with the application of an anti-staphylococcal agent leads to decolonization at other body sites and can prevent infection in surgical and dialysis patients, suggesting a causal relationship between nasal carriage and infection [32,33]. Our results show that up to $79 \%$ of $\mathrm{AD}$ patients carried the same spa-type in the nose and skin, indicating clonal colonization within individual patients and a possible role for endogenous $S$. aureus transmission from nose to skin and/or vice versa. These data form an important lead for future intervention studies investigating the effect of nasal S. aureus eradication on skin colonization patterns and their role in current treatment guidelines.

A large proportion of patients were persistent $S$. aureus carriers in the nose (76\%) and on the skin (58\%). There is no general definition for persistent and intermittent skin carriers or consensus on how many cultures should be taken from a skin site to define these groups [7, 31]. Studies on non-AD patients suggest that persistent carriers are colonized by a single strain in the nasal cavity over time and have a higher $S$. aureus load, with an increased risk of infection [7]. Literature on this subject in $\mathrm{AD}$ patients is scarce and include low patient numbers, but seem to confirm these findings $[13,14,17]$. Due to the absence of a general definition on carriership, the comparison between studies investigating $S$. aureus colonization patterns and temporal strain variation is difficult, as different sample frequencies and definitions have been used. Our study, with a strict 2-week follow-up and a large sample size, confirmed high rates of colonization by a single strain per individual in persistent carriers. We did not find a difference in disease severity between patients with and without temporal variation in colonizing $S$. aureus strain. However, more studies with larger populations should follow.

We found a significantly higher EASI score at baseline in patients positive for $S$. aureus in comparison to $S$. aureus-negative patients, which was not observed anymore after the 2-week run-in period with triamcinolone (T1). Furthermore, a significantly lower EASI score and S. aureus load were seen at $\mathrm{T} 1$ than at $\mathrm{T} 0$. The discrepancies in these results could be explained by the standardized TCS treatment, which is known to suppress inflammation in AD skin. Our hypothesis is that, due to a suppression of inflammation, the skin can recover, making it less prone to $S$. aureus overgrowth. Thus, the use of TCS disturbs the correlation at this time point. This was also proposed in a previous study, showing a reduction in S. aureus load and a correlation with disease severity after the use of standardized TCS treatment [34].

A major strength of this follow-up study is that all patients were sampled within the same interval of 2 weeks. Because treatment was similar in all patients, our results were not subject to differences in treatment regimens between participants. However, it is likely that the standardized use of TCS affected the disease severity and S. aureus 
density by inhibiting the inflammatory response, preventing subsequent damage to the skin and making it less prone to $S$. aureus overgrowth.

This study has several limitations. First, due to the lack of control group, we were not able to compare the presence of certain spa-types to a well-characterized control population. For this reason, we compared the spa-types in our study population with those in previous publications that described genetic populations in the general population and with data from "ridom SpaServer" [26]. Second, as mentioned before, due to the spa-typing technique used, we may have not detected changes in other genes that may be important in AD. Last, due to the high heterogeneity and low patient numbers per spa-type, statistical testing for differences between the genetic types was difficult. Larger studies using WGS will help to clarify the role of specific $S$. aureus genes rather than specific strains. Furthermore, the lack of association of specific spa-types with disease severity encourages further investigation of the potential role of other virulence factors.

In conclusion, our results show that the colonizing $S$. aureus strains are not specific for $\mathrm{AD}$ and might rather be caused by host-genetic and environmental factors. The majority of the AD patients were found to be persistent $S$. aureus carrier with identical spa-types in the nose and skin. The results of this study underline the relevance of investigating the clinical benefit of anti-staphylococcal treatment in $\mathrm{AD}$ patients that are persistently colonized with S. aureus.

\section{Key Message}

The skin and the nose are mostly colonized by a single strain of S. aureus. It is clinically relevant to investigate the benefit of antistaphylococcal treatment in persistent $S$. aureus carriers.

\section{Acknowledgement}

This study is part of the Academic Center of Excellences of Erasmus MC University Medical Center Rotterdam-Sophia Children's Hospital, Rotterdam, The Netherlands: Allergic diseases Atopic disorders and mast cell associated diseases and The Immunological Diseases Center. The authors gratefully thank all patients who participated in the study.

\section{Statement of Ethics}

This research was conducted ethically in accordance with the World Medical Association Declaration of Helsinki. Study procedures were reviewed and approved by the Medical Ethics Committee of the Erasmus MC University Medical Center Rotterdam, the Netherlands (MEC-2016-233). Signed informed consent was obtained from all participants.

\section{Conflict of Interest Statement}

The authors have no conflicts of interest to declare.

\section{Funding Sources}

The Department of Dermatology of the Erasmus MC University Medical Center Rotterdam received an unrestricted grant from Micreos Human Health, The Netherlands.

\section{Author Contributions}

S.G.M.A.P., J.E.E.T., J.W., and L.M.P. designed the study. M.M.F.M., J.E.E.T., and J.W. collected all the data. B.L.H. supervised the bacterial semi-quantitative culture and storage of colonies. M.C.V. and C.H.W.K. supervised the spa-typing. M.M.F.M., C.H.W.K., and L.M.P. performed the data analysis. M.M.F.M. wrote the manuscript. L.M.P. supervised the study. All authors critically commented on the manuscript.

\section{References}

1 Odhiambo JA, Williams HC, Clayton TO, Robertson CF, Asher MI; ISAAC Phase Three Study Group. Global variations in prevalence of eczema symptoms in children from ISAAC Phase Three. J Allergy Clin Immunol. 2009 Dec;124(6):1251-8.e23.

2 Weidinger S, Beck LA, Bieber T, Kabashima $\mathrm{K}$, Irvine AD. Atopic dermatitis. Nat Rev Dis Primers. 2018 Jun;4(1):1.

3 Totté JE, van der Feltz WT, Hennekam M, van Belkum A, van Zuuren EJ, Pasmans SG. Prevalence and odds of Staphylococcus aureus carriage in atopic dermatitis: a systematic review and meta-analysis. Br J Dermatol. 2016 Oct;175(4):687-95.
4 Guzik TJ, Bzowska M, Kasprowicz A, Czerniawska-Mysik G, Wójcik K, Szmyd D, et al. Persistent skin colonization with Staphylococcus aureus in atopic dermatitis: relationship to clinical and immunological parameters. Clin Exp Allergy. 2005 Apr;35(4):44855.

5 Tauber M, Balica S, Hsu CY, Jean-Decoster C, Lauze C, Redoules D, et al. Staphylococcus aureus density on lesional and nonlesional skin is strongly associated with disease severity in atopic dermatitis. J Allergy Clin Immunol. 2016 Apr;137(4):1272-1274.e3.
6 Higaki S, Morohashi M, Yamagishi T, Hasegawa Y. Comparative study of staphylococci from the skin of atopic dermatitis patients and from healthy subjects. Int J Dermatol. 1999 Apr;38(4):265-9.

7 Wertheim HF, Melles DC, Vos MC, van Leeuwen W, van Belkum A, Verbrugh HA, et al. The role of nasal carriage in Staphylococcus aureus infections. Lancet Infect Dis. 2005 Dec;5(12):751-62.

8 Toshkova K, Annemüller C, Akineden O, Lämmler C. The significance of nasal carriage of Staphylococcus aureus as risk factor for human skin infections. FEMS Microbiol Lett. 2001 Aug;202(1):17-24. 
9 Kluytmans J, van Belkum A, Verbrugh H. Nasal carriage of Staphylococcus aureus: epidemiology, underlying mechanisms, and associated risks. Clin Microbiol Rev. 1997 Jul;10(3): 505-20.

10 Kong HH, Oh J, Deming C, Conlan S, Grice EA, Beatson MA, et al.; NISC Comparative Sequence Program. Temporal shifts in the skin microbiome associated with disease flares and treatment in children with atopic dermatitis. Genome Res. 2012 May;22(5): 850-9.

11 Kuroda M, Ohta T, Uchiyama I, Baba T, Yuzawa H, Kobayashi I, et al. Whole genome sequencing of meticillin-resistant Staphylococcus aureus. Lancet. 2001 Apr;357(9264): 1225-40.

12 Byrd AL, Deming C, Cassidy SK, Harrison OJ, Ng WI, Conlan S, et al.; NISC Comparative Sequencing Program. Staphylococcus aureus and Staphylococcus epidermidis strain diversity underlying pediatric atopic dermatitis. Sci Transl Med. 2017 Jul;9(397):eaal4651.

13 Lomholt H, Andersen KE, Kilian M. Staphylococcus aureus clonal dynamics and virulence factors in children with atopic dermatitis. J Invest Dermatol. 2005 Nov;125(5):97782.

14 Clausen ML, Edslev SM, Nørreslet LB, Sørensen JA, Andersen PS, Agner T. Temporal variation of Staphylococcus aureus clonal complexes in atopic dermatitis: a follow-up study. Br J Dermatol. 2019 Jan;180(1):181-6.

15 Narukawa M, Yasuoka A, Note R, Funada H. Sequence-based spa typing as a rapid screening method for the areal and nosocomial outbreaks of MRSA. Tohoku J Exp Med. 2009 Jul; 218(3):207-13.

16 Hallin M, Friedrich AW, Struelens MJ. spa typing for epidemiological surveillance of Staphylococcus aureus. Methods Mol Biol. 2009;551:189-202.

17 Alsterholm M, Strömbeck L, Ljung A, Karami N, Widjestam J, Gillstedt M, et al. Variation in Staphylococcus aureus Colonization in Relation to Disease Severity in Adults with Atopic Dermatitis during a Five-month Follow-up. Acta Derm Venereol. 2017 Jul;97(7): $802-7$.
18 Totté J, de Wit J, Pardo L, Schuren F, van Doorn M, Pasmans S. Targeted anti-staphylococcal therapy with endolysins in atopic dermatitis and the effect on steroid use, disease severity and the microbiome: study protocol for a randomized controlled trial (MAAS trial). Trials. 2017 Aug; 18(1):404.

19 de Wit J, Totté JE, van Mierlo MM, van Veldhuizen J, van Doorn MB, Schuren FH, et al. Endolysin treatment against Staphylococcus aureus in adults with atopic dermatitis: A randomized controlled trial. J Allergy Clin Immunol. 2019 Sep;144(3):860-3.

20 Leshem YA, Hajar T, Hanifin JM, Simpson EL. What the Eczema Area and Severity Index score tells us about the severity of atopic dermatitis: an interpretability study. Br J Dermatol. 2015;172(5):1353-7.

21 Kim DW, Park JY, Park KD, Kim TH, Lee WJ, Lee SJ, et al. Are there predominant strains and toxins of Staphylococcus aureus in atopic dermatitis patients? Genotypic characterization and toxin determination of S. aureus isolated in adolescent and adult patients with atopic dermatitis. J Dermatol. 2009 Feb;36(2): 75-81.

22 Yeung M, Balma-Mena A, Shear N, Simor A, Pope E, Walsh S, et al. Identification of major clonal complexes and toxin producing strains among Staphylococcus aureus associated with atopic dermatitis. Microbes Infect. 2011 Feb;13(2):189-97.

23 Holtfreter S, Grumann D, Balau V, Barwich A, Kolata J, Goehler A, et al. Molecular Epidemiology of Staphylococcus aureus in the General Population in Northeast Germany: Results of the Study of Health in Pomerania (SHIP-TREND-0). J Clin Microbiol. 2016 Nov;54(11):2774-85.

24 Sangvik M, Olsen RS, Olsen K, Simonsen GS, Furberg AS, Sollid JU. Age- and gender-associated Staphylococcus aureus spa types found among nasal carriers in a general population: the Tromso Staph and Skin Study. J Clin Microbiol. 2011 Dec;49(12):4213-8.

25 Becker K, Schaumburg F, Fegeler C, Friedrich AW, Köck R; Prevalence of Multiresistant Microorganisms PMM Study. Staphylococcus aureus from the German general population is highly diverse. Int J Med Microbiol. 2017 Jan;307(1):21-7.

26 Ridom GmbH. Ridom SpaServer [updated December 2005]. Available from: https:// www.spaserver.ridom.de/.
27 Asadollahi P, Farahani NN, Mirzaii M, Khoramrooz SS, van Belkum A, Asadollahi K, et al. Distribution of the Most Prevalent Spa Types among Clinical Isolates of MethicillinResistant and -Susceptible Staphylococcus aureus around the World: A Review. Front Microbiol. 2018 Feb;9:163.

28 SenGupta DJ, Cummings LA, Hoogestraat DR, Butler-Wu SM, Shendure J, Cookson BT, et al. Whole-genome sequencing for high-resolution investigation of methicillin-resistant Staphylococcus aureus epidemiology and genome plasticity. J Clin Microbiol. 2014 Aug; 52(8):2787-96.

29 Koreen L, Ramaswamy SV, Graviss EA, Naidich S, Musser JM, Kreiswirth BN. spa typing method for discriminating among Staphylococcus aureus isolates: implications for use of a single marker to detect genetic micro- and macrovariation. J Clin Microbiol. 2004 Feb; 42(2):792-9.

30 Sivaraman K, Venkataraman N, Cole AM. Staphylococcus aureus nasal carriage and its contributing factors. Future Microbiol. 2009 Oct;4(8):999-1008.

31 Sakr A, Brégeon F, Mège JL, Rolain JM, Blin O. Staphylococcus aureus Nasal Colonization: An Update on Mechanisms, Epidemiology, Risk Factors, and Subsequent Infections. Front Microbiol. 2018 Oct;9:2419.

32 Kluytmans JA, Wertheim HF. Nasal carriage of Staphylococcus aureus and prevention of nosocomial infections. Infection. $2005 \mathrm{Feb}$; 33(1):3-8.

33 Peng HM, Wang LC, Zhai JL, Weng XS, Feng $B$, Wang W. Effectiveness of preoperative decolonization with nasal povidone iodine in Chinese patients undergoing elective orthopedic surgery: a prospective cross-sectional study. Braz J Med Biol Res. 2017 Dec; 51(2):e6736.

34 Gonzalez ME, Schaffer JV, Orlow SJ, Gao Z, Li H, Alekseyenko AV, et al. Cutaneous microbiome effects of fluticasone propionate cream and adjunctive bleach baths in childhood atopic dermatitis. J Am Acad Dermatol. 2016 Sep;75(3):481-493. e8.

35 Brüssow H. Turning the inside out: the microbiology of atopic dermatitis. Environ Microbiol. 2016 Jul;18(7):2089-102. 